\title{
Skin nodules in a 16-year-old adolescent with acute lymphoblastic leukemia
}

\author{
Amanda Ruth MD ${ }^{1}$, Hernan Sabio MD¹, Thomas W McLean MD¹, Cyrus Manavi MD², \\ Elizabeth L Palavecino MD², Avinash K Shetty MD ${ }^{1}$
}

\begin{abstract}
A 16-year-old girl with B-lineage acute lymphoblastic leukemia was hospitalized for management of fever and neutropenia. She was empirically treated with intravenous vancomycin and ceftazidime. Her fever persisted, and on the seventh day of hospitalization, she developed a painful generalized erythematous rash. There was no history of trauma, infectious contacts, travel, gardening or pet exposures.

On physical examination, she was febrile $\left(39.2^{\circ} \mathrm{C}\right)$, but appeared well with no apparent distress. A skin examination revealed a rash that consisted of numerous violaceous papules, nodules and plaques on her trunk, abdomen and lower extremities. The lesions were surrounded by a $1 \mathrm{~cm}$ area of cellulitis, and some had necrotic centres (Figure 1). Otolaryngological evaluation revealed a necrotic nasal septum. Her abdominal examination was significant for hepatosplenomegaly. The rest of the examination was unremarkable.

Laboratory data were significant for a white blood cell count of $0.4 \times 10^{9} / \mathrm{L}$, hemoglobin level of $84 \mathrm{~g} / \mathrm{L}$ and a platelet count of $21 \times 10^{9} / \mathrm{L}$. Renal function and liver enzymes were normal; blood and urine cultures were negative for bacteria and fungi. A computed tomography scan of the chest with contrast revealed bilateral pulmonary nodules. A
\end{abstract}

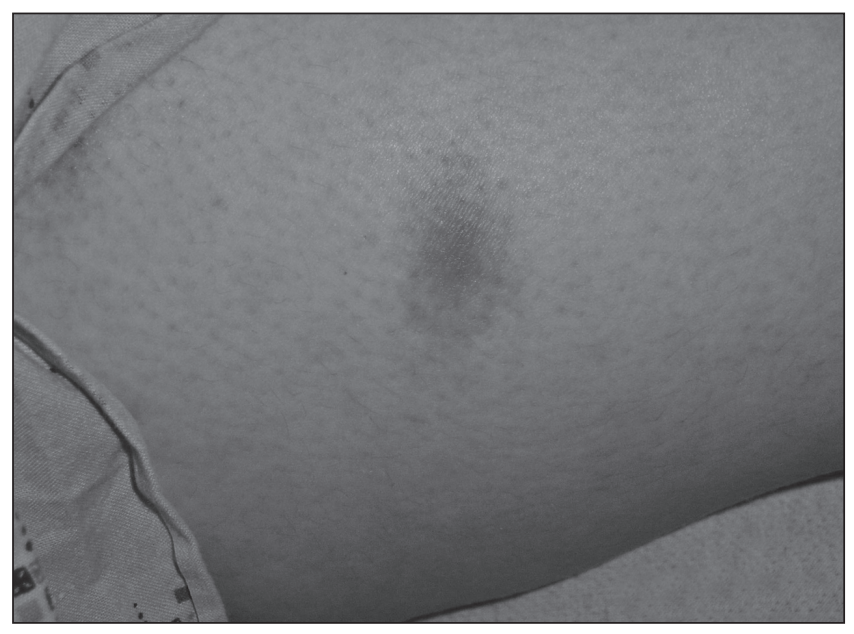

Figure 1) Skin lesion on thigh cutaneous punch biopsy was performed, and specimens were submitted for culture and histopathology (Figures 2 and 3).

What is the diagnosis?

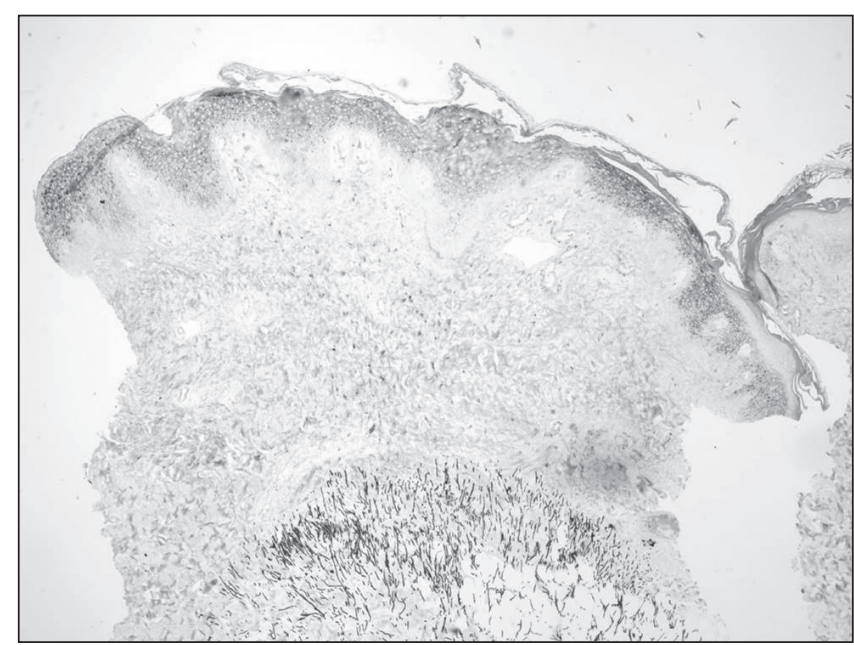

Figure 2) Deep reticular dermis. Gomori methenamine silver stain, original magnification $\times 10$

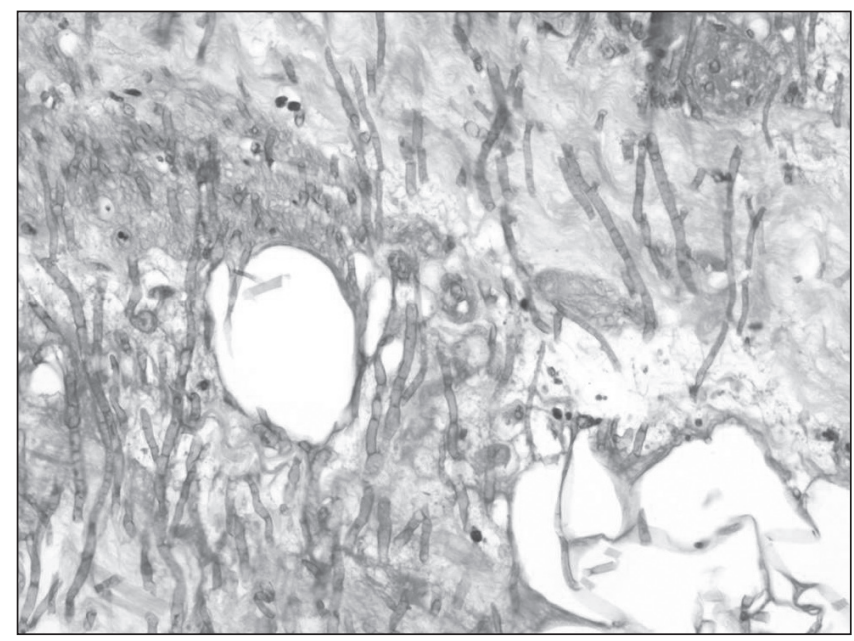

Figure 3) High power view of periodic acid-Schiff stain

\footnotetext{
${ }^{1}$ Department of Pediatrics; ${ }^{2}$ Department of Pathology, Wake Forest University Health Sciences, Brenner Children's Hospital, Winston-Salem, North Carolina, USA

Correspondence: Dr Avinash K Shetty, Department of Pediatrics, Wake Forest University Health Sciences, Medical Center Boulevard,

Winston-Salem, North Carolina 27157, USA. Telephone 336-716-2740, fax 336-716-7100, e-mail ashetty@wfubmc.edu

Received and accepted for publication May 26, 2008
} 


\section{DIAGNOSIS}

The histopathological specimen using periodic acid-Schiff stain (original magnification $\times 40$ ) and Grocott's methenamine silver stain (original magnification $\times 10$ ) showed heavy growth of branching septate hyphae in the deep dermis and cutis suggesting an invasive fungal infection. On tissue culture, the fungus was identified as Fusarium species. A diagnosis of invasive Fusarium species infection was made, and treatment with intravenous amphotericin $B$ was initiated. The patient also underwent surgical debridement and removal of infected nasal turbinates bilaterally. The patient was treated with intravenous amphotericin B for three weeks, and was then discharged on oral voriconazole to complete a three-month course of antifungal therapy. She responded well and improved clinically on oral voriconazole, with resolution of cutaneous and pulmonary lesions. She died six months later due to sepsis caused by Pseudomonas aeruginosa.

\section{DISCUSSION}

Fusarium species are filamentous fungi that are ubiquitous in the environment. In immunocompetent hosts, onychomycosis and keratitis are the most common infections. However, in leukemia patients and stem cell transplant recipients, disseminated infection caused by this angioinvasive opportunistic pathogen is serious and life-threatening, with a mortality rate of $50 \%$ to $70 \%$ (1). The frequency of invasive mycoses, including Fusarium species infection, has increased significantly over the past two decades in patients with hematological malignancies. This increase is directly related to the increased use of aggressive chemotherapy, and blood and stem cell transplantation (1). Dissemination occurs via the blood stream after entry through the lungs or skin. Risk factors for infection include prolonged neutropenia, intensive chemotherapy, corticosteroid use and graft-versus-host disease.

The most common presentation of invasive fusariosis is fever refractory to antibiotic therapy. Disseminated Fusarium infection often presents with painful skin nodules with or without lung or sinus involvement (1). Skin lesions may have an ecthyma gangrenosum-like or target appearance, or may present as multiple subcutaneous nodules (2). Pulmonary fusariosis usually presents with nonspecific respiratory symptoms such as cough and dyspnea, and only rarely hemoptysis. On chest radiography, nonspecific infiltrates are most frequently noted; less common findings include nodular and cavitary lesions.

Definitive diagnosis requires isolation of Fusarium from a clinical specimen. Positive blood cultures are noted in $40 \%$ of disseminated cases (1). Unlike aspergillosis, skin lesions are more frequently encountered with disseminated fusariosis, and are often the only source of diagnostic information. Histopathological findings include hyaline branching septate hyphae invading the skin and blood vessels, sometimes with thrombosis and necrosis (3). However, culture identification remains crucial because Fusarium species is histopathologically similar to many other fungi, particularly Aspergillus and Acremonium species. In addition, the two species are treated differently and have a different prognosis.

Amphotericin B or its lipid formulations remain the mainstay of treatment for invasive fusariosis. Recent reports (4) indicate that concurrent treatment with amphotericin B and newer antifungal agents, such as voriconazole or caspofungin, lead to a better outcome. In refractory cases, oral posaconazole may be beneficial as salvage treatment (5). For many patients, resolution of infection is only seen with neutrophil recovery; therefore, the addition of granulocyte colony-stimulating factor to the antifungal treatment regimen may be useful when clinically indicated. Because disseminated fusariosis carries a high mortality rate, there needs to be a high index of clinical suspicion when physicians encounter skin lesions in neutropenic immunocompromised patients.

\section{REFERENCES}

1. Nucci M, Anaissie E. Fusarium infections in immunocompromised patients. Clin Microbiol Rev 2007;20:695-704.

2. Chi CC, Wang SH. Disseminated cutaneous Fusarium moniliforme infections in a leukemic child. Int J Dermatol 2007;46:487-9.

3. Vagace JM, Sanz-Rodriguez C, Casado MS, et al. Resolution of disseminated fusariosis in a child with acute leukemia treated with combined antifungal therapy: A case report. BMC Infect Dis 2007;10:40.

4. Ho DY, Lee JD, Rosso F, Montoya JG. Treating disseminated fusariosis: Amphotericin B, voriconazole or both? Mycoses 2007;50:227-31.

5. Petrikkos G, Skiada A. Recent advances in antifungal chemotherapy. Int J Antimicrob Agents 2007;30:108-17. 


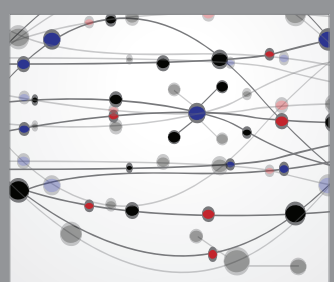

The Scientific World Journal
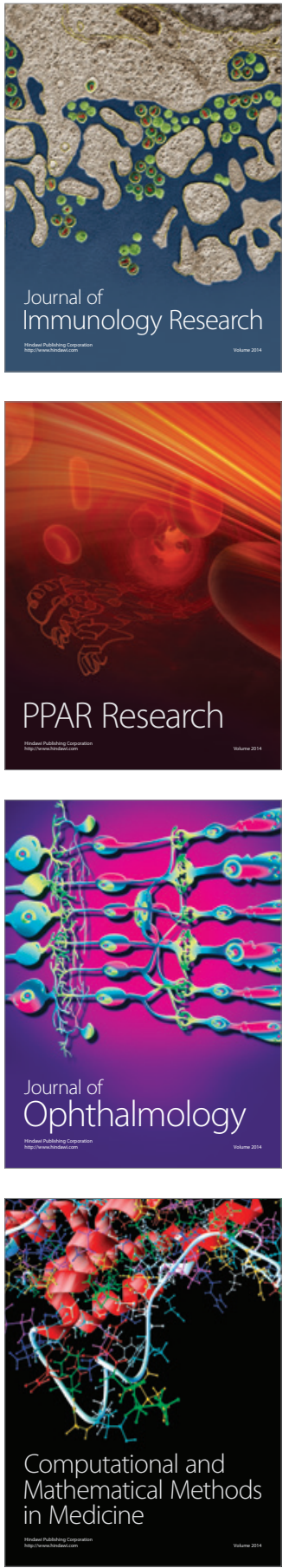

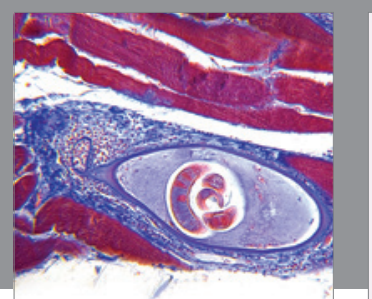

Gastroenterology Research and Practice

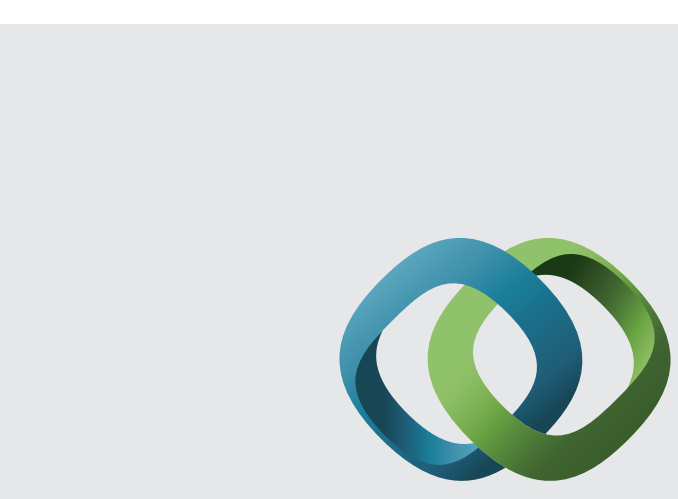

\section{Hindawi}

Submit your manuscripts at

http://www.hindawi.com
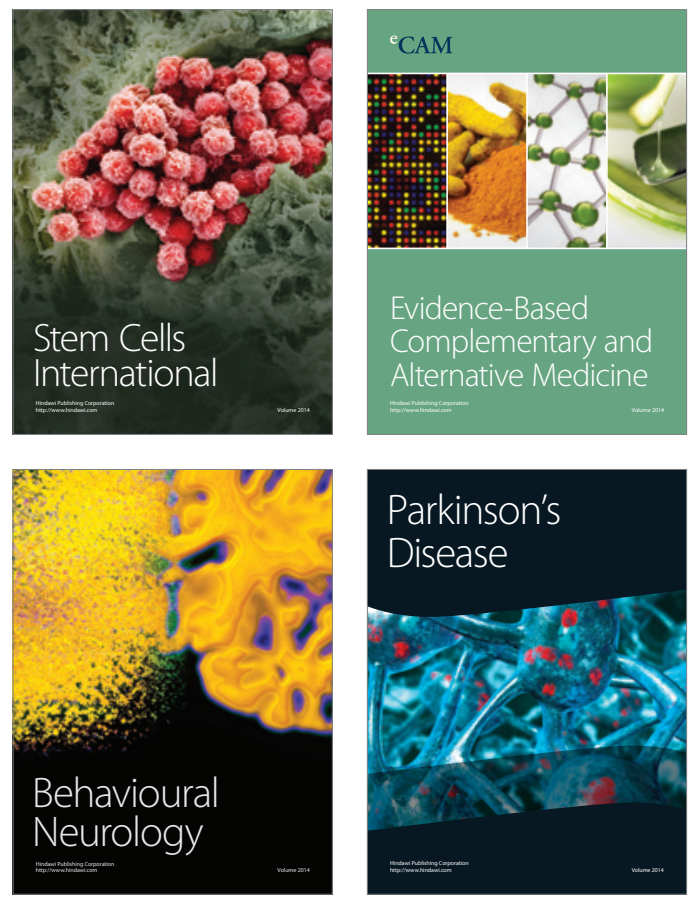
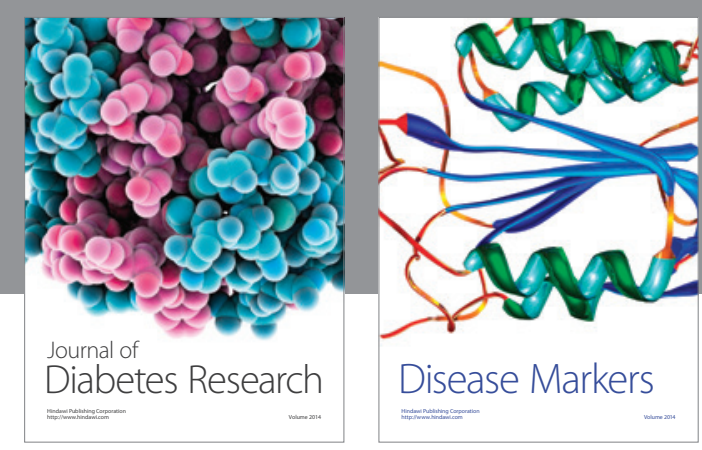

Disease Markers
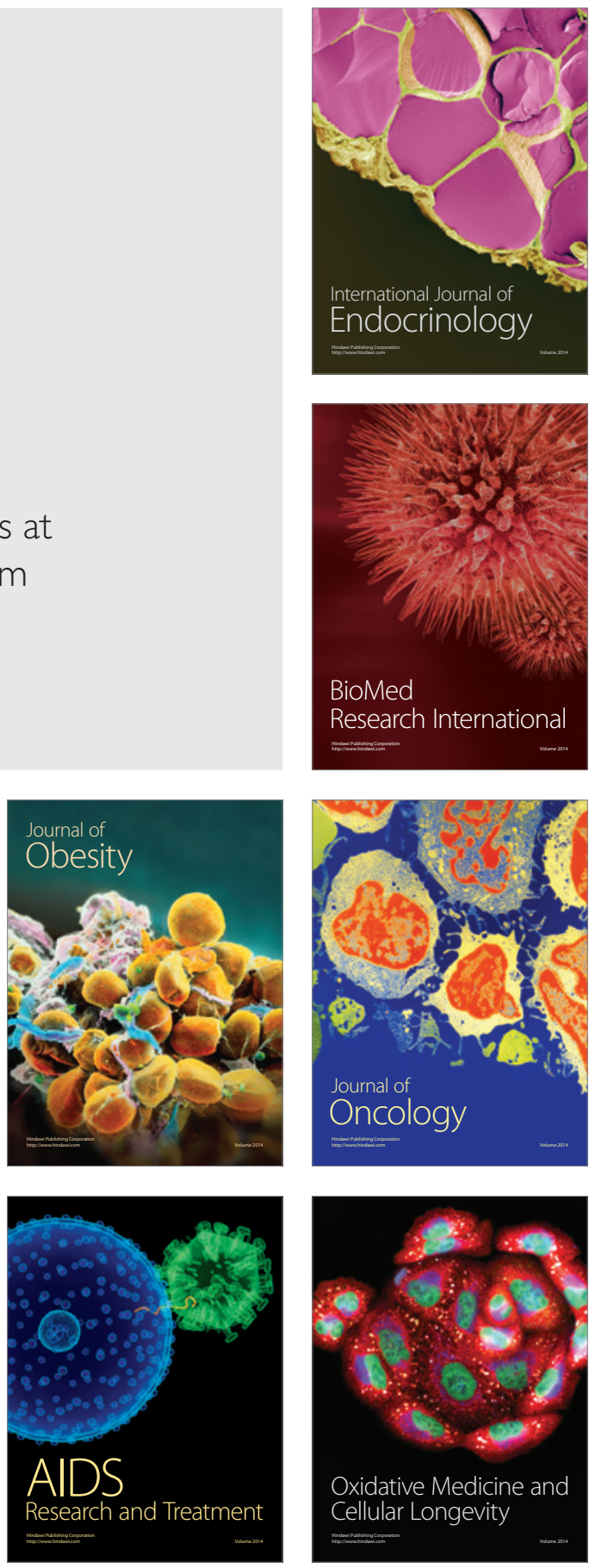